\title{
Estimation of Local Mean Population Densities of Japanese Beetle Grubs (Scarabaeidae: Coleoptera)
}

\author{
D. DALTHORP, J. NYROP, AND M. VILLANI
}

Department of Entomology, Cornell University, NYSAES, Geneva, NY 14456

\begin{abstract}
Environ. Entomol. 28(2): 255-265 (1999)
ABSTRACT Insect populations tend to be patchy in distribution. Even when the mean population density is low, there may be local patches with high densities. As a result, estimates of mean populations may provide little information about the size or intensity of local patches within the sampled area. We compared the following 3 methods of estimating local population densities of insects: (1) with moving averages, a local mean population density is estimated as the mean of samples taken within a given radius of a central point, (2) with inverse distances, local means are estimated as weighted averages of samples; each sample is given a weight proportional to a power of the reciprocal of its distance from the center of the region for which the mean is to be estimated, (3) kriging is a geostatistical algorithm for estimating local means as weighted averages of samples. Weighting is based on the spatial covariance of the samples, or the degree to which samples that are near to each other are related. The first 2 methods are relatively easy to calculate but were unreliable when used with standard parameters to estimate local Japanese beetle grub densities. When an optimum radius was used with moving averages and an optimum exponent was used with inverse distances, the advantage of ease of calculation was lost, yet both methods were still inferior to kriging in providing accurate estimates of local means.
\end{abstract}

KEY WORDS Popillia japonica, larvae, spatial statistics, inverse distance, kriging, dispersion

INSECT POPULATION DENSITIES are commonly estimated as the mean of a set of sample observations. This approach works well for estimating overall mean populations, but more sophisticated approaches to estimating local means are needed when identification of patches with high or low density is desired. Because the distribution of agricultural pests is often patchy, pest populations may be high enough in some parts of a field to damage a crop, whereas other parts of the field remain virtually pest-free (Nyrop et al. 1995; Weisz et al. 1995a, b). Targeting management tactics such as application of insecticides to the parts of the field that harbor high pest populations may retard the development of resistance in the pest population and conserve natural enemies (e.g., Midgarden et al. 1997). Such precision management of insect pests requires reliable methods for estimating mean population densities in the smallest areas or blocks in which significant damage could occur. When a single sample observation provides a good estimate of mean density within a block surrounding the sample, or when many samples can be taken within the block so that a reliable estimate of the mean density can be made by taking the average of the observations, the problem of estimating the local means is solved. These solutions are not feasible when individual sample observations are poor estimators of the local population means, and it is not feasible to take several samples within each block. In such cases, it may still be possible to estimate local means reliably by taking advantage of spatial autocorrelation, or the tendency of neighboring samples to have similar values.

We were faced with this problem of estimating local means for larvae of Japanese beetle, Popillia japonica Newman, on golf course fairways. The fairways are mixed plantings of turfgrass, each covering $6,000-16,000 \mathrm{~m}^{2}$. Golf courses normally contain 18 fairways, or some $200,000 \mathrm{~m}^{2}$ of intensely managed turf. In the eastern United States, Japanese beetle larvae are common pests on these plantings. Visible grub damage can occur in block of turf as little as $45-90 \mathrm{~m}^{2}$. Given the highly variable distribution of Japanese beetle larvae in these turf plantings, reliable estimates of local population densities could be useful management aids.

A golf course may contain $>3,000$ blocks in which to estimate local means, so it may not be feasible to collect several samples in each block. In addition, the small size of each sample $\left(0.008 \mathrm{~m}^{2}\right)$ precludes it from being a precise estimate of a local mean. We compared several methods of taking advantage of spatial covariance of population density to use information from nearby blocks to estimate the mean of a given block. Each method estimates a block mean as a weighted average of samples in and around the block. Weights decrease as a function of distance from the center of the block. The methods differ only in how those weights are assigned. The 3 methods of estimating local means were compared on the basis of effectiveness (or ability to make correct estimates) and stabil- 
Table 1. Summary of sample counts of Japanese beetle grubs on 2 golf courses for each of 2 yr

\begin{tabular}{|c|c|c|c|c|c|c|c|}
\hline Year & Golf course & Fairway & Rows $^{a}$ & Columns $^{b}$ & $n$ & Mean $^{c}$ & Variance \\
\hline \multirow[t]{11}{*}{1995} & Tuscarora & T1895 & 11 & 9 & 82 & 0.805 & 1.122 \\
\hline & & T1095 & 15 & 9 & 130 & 1.023 & 1.961 \\
\hline & & T1195* & 15 & 9 & 134 & 1.709 & 3.546 \\
\hline & & T1295 & 16 & 11 & 171 & 1.047 & 2.621 \\
\hline & & T1395* & 10 & 12 & 106 & 1.085 & 1.621 \\
\hline & Silver & S0895* & 10 & 10 & 81 & 1.062 & 2.734 \\
\hline & Creek & & & & & & \\
\hline & & S1195* & 12 & 21 & 236 & 0.822 & 1.960 \\
\hline & & S1395* & 14 & 11 & 146 & 1.356 & 3.169 \\
\hline & & S1695* & 13 & 9 & 117 & 0.872 & 1.544 \\
\hline & Average & & 12.9 & 11.2 & 133.7 & 1.087 & 2.253 \\
\hline \multirow[t]{11}{*}{1996} & Tuscarora & T1896* & 21 & 9 & 143 & 0.664 & 1.239 \\
\hline & & T1096* & 29 & 9 & 255 & 0.808 & 1.463 \\
\hline & & T1196* & 29 & 9 & 254 & 1.280 & 2.732 \\
\hline & & T1296* & 31 & 11 & 326 & 0.546 & 0.907 \\
\hline & & T1396* & 19 & 12 & 201 & 0.866 & 1.327 \\
\hline & Silver & S0896 & 19 & 10 & 153 & 0.275 & 0.398 \\
\hline & Creek & & & & & & \\
\hline & & S1196 & 23 & 21 & 451 & 0.188 & 0.291 \\
\hline & & S1396 & 27 & 11 & 280 & 0.154 & 0.166 \\
\hline & & S1696* & 25 & 9 & 225 & 0.231 & 0.384 \\
\hline & Average & & 24.8 & 11.2 & 254.2 & 0.557 & 0.990 \\
\hline
\end{tabular}

Fairways for which detailed spatial analyses were performed are indicated by asterisks.

${ }^{a}$ Number of rows of samples taken across fairway.

${ }^{b}$ Number of columns of samples taken along fairway.

${ }^{c}$ Average number of grubs per sample.

ity (or ability to give consistent results), as discussed below.

Moving Averages. With the method of moving averages, a local or block mean is estimated as the average of all samples within a given radius of the center of the block. Moving averages has the advantage that local means are easy to calculate. Disadvantages of this method are that it is difficult to determine the most appropriate radius for smoothing, and the implicit model of spatial covariance may not be correct, in which case good estimates of local means would not be obtained.

Inverse Distances. Isaaks and Srivastava (1989) provide an excellent discussion of the commonly used inverse distance method for spatial interpolation and map generation. However, the method must be modified if it is to be used for estimating local means. The modified method is discussed below, and details of the modifications are discussed in the Appendix 1 .

Local means are estimated as weighted averages of the samples taken on a fairway. Weights assigned to sample points are proportional to a power of the inverse of the distance from the sample to the center of the block. Points far from the block thus receive little weight, and points close to the block receive relatively high weights. The weights are normalized so that they sum to 1 . The estimate of the block mean is the weighted average of all points on the fairway, viz.

$$
\hat{\mu}_{j}=\frac{w_{j} z_{j}+\sum_{i \neq j} \frac{1}{d_{i}{ }^{p}} z_{i}}{w_{j}+\sum_{i \neq j} \frac{1}{d_{i}{ }^{p}}},
$$

where $\hat{\mu}$, is the estimate of the mean population density in block $j, d_{\mathrm{i}}$ is the distance from the center of the block to sample $i, z_{\mathrm{i}}$ is the number of grubs observed in sample $i$, and $w_{\mathrm{j}}$ is the weight assigned to the sample at the center of the block (see Appendix and Table 2). The gradual decline in weights with distance is intuitively satisfying, yet the weights are still relatively easy to calculate. A priori disadvantages are that the implicit model of spatial covariance (or how the weights decline) is not very flexible, and optimization of the exponent $p$ requires considerable computation.

Block Kriging. With block kriging, local means are again estimated as a weighted average of the samples (Isaaks and Srivastava 1989, Deutsch and Journel 1992). Weights are based on an explicit model of the spatial covariance of the data. We used the covariogram to quantify the covariance (Cressie 1993, Kaluzny et al. 1996). For each fairway, 2 different covariance models were used: omnidirectional and directional. Omnidirectional models assume that the covariance is strictly a function of separation distance or lag. With directional models, covariance is modeled as a function of both the lag distance and direction. Directional models are useful when populations are more continuous in 1 direction than another, as commonly occurs when an environmental gradient changes more rapidly in one direction than another. The advantage of this method is that the model of spatial covariance is explicit and flexible, resulting in relatively reliable estimates of local means. The main disadvantage is that the method does require explicit modeling of the spatial covariance.

Comparison of the Methods. Effectiveness. The effectiveness of estimation methods can be quantified 
by comparing estimates to true values using crossvalidation. In cross-validation, actual data points are dropped one at a time and reestimated from the remaining data. Thus, the sample count at the center of a block is ignored when calculating the cross-validation estimate for that block. Once a datum has been reestimated using an estimation method, it is put back into the data set and the procedure is repeated for the next datum (Deutsch and Journel 1992). In this way a cross-validation data set is constructed for each estimation method with 1 cross-validation estimate corresponding to each point in the data set. Cross-validation estimates are subtracted from the raw data values to obtain the cross-validation errors. The crossvalidation errors measure how well a given method estimates means at 'unsampled' locations. Thus, an effective method should have relatively small crossvalidation errors.

Stability. Stability of an estimation method is defined in terms of the similarity of its interpretation of local means for different smoothing parameters (e.g., the radius for moving averages). Different smoothing parameter values result in unique topographical maps or surfaces of block means, with peaks of high density and valleys of low density. For example, with decreasing inverse distance exponent or increasing moving average radius, each estimated local mean approaches the overall sample mean, so the density surface becomes smoother. Conversely, for large inverse distance exponent or small moving average radius, the density surface is relatively rough.

With fortuitous circumstances in some applications, choice of a smoothing parameter within a wide range of values does not significantly affect the interpretation of the data (e.g., Weisz et al. 1995a, b). In such situations, a carefully selected value may be chosen as a standard value for widespread use. However, in other situations the interpretation of data can change substantially with small changes in parameter values, making the estimation method unreliable if a standard smoothing parameter is used.

A stability index can be used to quantify the differences in the topographies of density surfaces resulting from small changes in smoothing parameter values. This index then can be used as a relative measure of stablity to compare estimation methods for a single set of data. The stability index also can be used to determine when instability precludes the use of a standard inverse distance exponent or moving average radius for a collection of data sets.

\section{Materials and Methods}

Sampling. Five fairways at Tuscarora Country Club in Camillus, NY, and 4 at Silver Creek Country Club in Waterloo, NY, were sampled for 2 nd and 3rd instars of Japanese beetle in late August and early September 1995 and 1996 . The fairways were $\approx 200 \mathrm{~m}$ long and $40 \mathrm{~m}$ wide. All were irrigated, and normal applications of fertilizers, herbicides, and fungicides were applied

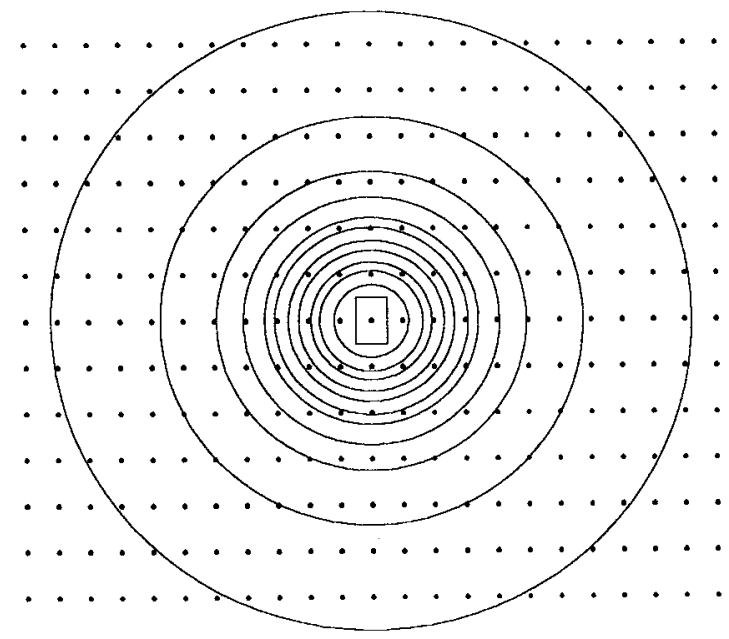

Fig. 1. Smoothing radii used in calculation of moving averages.

as needed. Insecticides were not applied in the spring or summer before each year's sampling, but bendiocarb was applied to the Tuscarora fairways after sampling.

Circular soil cores $10 \mathrm{~cm}$ in diameter and $\approx 10 \mathrm{~cm}$. deep were dug using golf course cup cutters. The cores were broken apart by hand, and the Japanese beetle grubs found in each core were counted and recorded along with the location of the sample. Samples were taken in 4.6-m intervals along transects (rows) across each fairway. In 1995, rows were separated by $14 \mathrm{~m}$ along the length of each fairway; the sampling intensity was doubled in 1996 by decreasing the distance between rows to $7 \mathrm{~m}$ (Table 1 ).

Estimation of Local Means. Local mean densities were estimated for rectangular blocks centered at each sample point. In 1995 fairways, the blocks measured 4.6 by $14 \mathrm{~m}$, and in 1996 the blocks were 4.6 by $7 \mathrm{~m}$. Mean population densities were estimated for each block by 3 methods-moving averages, inverse distances, and block kriging. For moving averages, estimates were made for all sampled blocks with smoothing radii of $0,5.5,7.6,8.9,10.7,12.2,14.2,15.7$, 18.8, 22.7, 31, and $47 \mathrm{~m}$ as indicated in Fig. 1. As discussed below, we used cross validation to determine the optimum radii for each fairway. For inverse distances, estimates were made for all sampled blocks, using inverse distance exponents of $P=0,0.5,0.75,1$, $1.25,1.5,1.75,2,2.5,3,3.5,4,4.5$, and 5 . An exponent of $P=0$ implies that all points receive the same weight, so estimates of all local means are equal to the overall mean. As $p \rightarrow \infty$, the estimates of block means approach the raw grub counts. As discussed below, we used cross validation to determine the optimum exponent for the various fairways. For block kriging, omnidirectional and directional covariance models were used to obtain omnidirectional and directional block kriging estimates of local means, respectively. 
Effectiveness of Estimation of Local Means. Crossvalidation estimates were made for each fairway for inverse distances with each of the exponents, for moving averages with each of the radii, and for both omnidirectional and directional kriging.

Quanitification of Cross-Validation Errors. The magnitude of the cross-validation errors for a given set of estimates was measured 2 ways - as the sum of squared errors (SSE), and as sums of absolute values of errors $(S A E)$. The following standardizations of SSE and SAE were then made:

mean squared error: $M S E=\frac{\sum\left(z_{i}^{*}-z_{i}\right)^{2}}{n}$,

mean absolute error: $M A E=\frac{\sum\left|z_{i}^{*}-z_{i}\right|}{n}$,

coefficient of determination: $R^{2}=\frac{S S T-S S E}{S S T}$,

relative absolute error $\left(\right.$ analog of $\left.\mathbf{R}^{2}\right)$ :

$$
A=\frac{S A T-S A E}{S A T},
$$

where $z_{\mathrm{i}}^{*}$ is the cross-validation estimate of sample $i$; $z_{i}$ is the number of grubs in sample $i ; n$ is the total number of samples on the fairway; and SST and SAT are total sums of squared and absolute errors (i.e., the SSE and $S A E$ for the null model, in which the overall mean is taken as the estimate for each local mean). Thus, $M S E$ and MAE measure the average magnitude of errors, and $R^{2}$ and $A$ are measures of the improvement a given model makes over the null model, with values of 0 indicating no improvement, 1 indicating a perfect model, and negative numbers indicating a poorer fit than than that of the null model.

Optimization. For each fairway, the moving average radius and inverse distance exponent that minimized the cross-validation MSE and MAE were selected as the optimum parameters. To make the final MSE-optimized and MAE-optimized estimates for a fairway, the moving average and inverse distance calculations were repeated using the optimum parameters with all the sample points on the fairway. Kriging estimates are based on models of the covariograms rather than on minimization of cross-validation errors, but the crossvalidation errors still were calculated for each fairway for comparison with the other methods.

Relative Effectiveness. To facilitate comparisons of the methods' effectiveness among fairways, the $R^{2}$ and $A$ values (equations. 4 and 5 ) for each method were standardized for each fairway by dividing by the $R^{2}$ and $A$ for directional kriging on that fairway. This is a measure of relative effectiveness based on a common scale for all fairways and methods. Thus, the relative effectiveness of directional kriging for both MSE and $M A E$ for each fairway is 1 , and the other methods are compared against this standard. For example, a relative MSE of 0.7 for a method implies that the method was $70 \%$ as effective as directional kriging at improving on the null model's MSE, or $R^{2}{ }_{\text {method }} / R^{2}{ }_{\text {directionalkriging }}=$
0.7. Because results are independent of which method is used as a standard, our choice of directional kriging was based solely on esthetic reasons.

Stability of the Methods. We defined stability of moving averages and inverse distances in terms of the changes in estimates of local means for small changes in moving average smoothing radius or inverse distance exponent.

Critical Range of Parameters. For each fairway, we calculated the optimum exponent for inverse distance and optimum radius for moving averages by selecting the values that resulted in the smallest cross-validation MSE and MAE. We then removed the highest and lowest values and defined the critical range of parameters to be the remaining interval. For example, the MSE-optimized inverse distance exponents for the twelve fairways were 1.25, 1.5 , 1.75, 2, 2, 2, 2, 2.25, 2.25, $2.25,2.5$, and 2.75. After removing the highest and lowest of these exponents, the truncated range is 1.52.5. This, then, was the critical range for MSE-optimized inverse distance. Similarly, we calculated the critical ranges for $M A E$-optimized inverse distance, $M S E$-optimized moving average, and MAE-optimized moving average. As defined, the critical range is an estimate of the range of optimum parameter values that were commonly observed in the field. A method that can be standardized reliably should give consistent results for any choice of parameter value in the critical range.

Stability Index. The degree of smoothness of the grub density surface is summarized by a statistic that we refer to as the estimated population variance, or the variance of the collection of estimated local means for a given fairway and method. A high population variance corresponds to a highly variable or rough surface, whereas a low population variance corresponds to a smooth surface. To quantify stability, we defined a stability index for a given fairway as the ratio of population variances resulting from the highest and lowest parameter values in the critical range. For example, the critical range for MSE-optimized inverse distance exponents is 1.5-2.5. The estimated population variances on T1896 for exponents 1.5 and 2.5 are 0.17 and 0.58 , respectively, so the stability index for MSE-optimized inverse distance on T1896 is $0.58 / 0.17=3.4$. By contrast, the critical range for MSE-optimized moving average radii is $10.7-18.8 \mathrm{~m}$. The estimated population variances on T1896 for radii 18.8 and 10.7 are 0.31 and 0.21 , respectively, giving a stability index of 1.5 . By this measure, the inverse distance method has a higher stability index value and is more unstable than moving averages on fairway T1896. We calculated stability indices for both inverse distances and moving averages optimized for MSE and MAE on all 12 fairways analyzed.

Critical Ratio and Quantification of the Practical Implications of the Stability Index. Because of space limitations, the nature and degree of the uncertainties captured by the stability index were analyzed in detail for only 1 fairway (T1896). Differences in grub density surfaces for parameters within the critical range for MSE-optimized inverse distance exponents and mov- 
ing average radii were plotted in wireframe graphs of estimated local mean populations. The wireframe graphs provide a visual summary of the practical importance of the stability index. Differences between the surfaces then were quantified via differences in the heights and extents of the peaks. The peaks were characterized by the upper percentiles (50th, 75th, 90th, 95th, 98th, and maximum) of the estimated local means on the fairway. These percentiles were then plotted as functions of the inverse distance exponent and moving average radius. Of particular interest were the differences in the estimates of each percentile for parameters within the critical range. To summarize such differences, we defined the critical ratio for a given method and percentile on a fairway as the ratio of the highest to the lowest estimate of the percentile for parameter values in the critical range. For example, the estimates for the 98 th percentile of block means on T1896 for inverse distance exponents of 1.5 and 2.5 (spanning the critical parameter range) are 1.58 and 2.73 , respectively. Therefore, the 98 th percentile critical ratio for inverse distances on T1896 is 2.73/1.58 = 1.73 , implying that within the critical range, estimates of the 98 th percentile vary by a factor of 1.73 . For each fairway the critical ratio was calculated for inverse distances and moving averages, each optimized for $M S E$ and MAE. Then for each method, the geometric mean critical ratio across fairways was calculated and plotted against percentile. We used the geometric mean whenever calculating an average of ratios or factors. The geometric mean considers $1 / 2$ and 2 to be equidistant from 1, an apt view when dealing with factors and ratios. This allows for easy comparison of the robustness of the methods' estimates of the heights of the peaks and how those estimates depend on relative grub population density.

Fairway Selection. Detailed spatial statistical analyses were performed on 12 of 18 fairways sampled. Not included were 2 fairways that lacked significant spatial covariance-S1396 had a mean grub density of 0.15 per sample, and the count distribution did not differ from Poisson ( $P=0.18$, chi-square test $)$; S0896 had a mean grub density of 0.27 per sample, and neither the variogram nor covariogram revealed any spatial covariance structure. Not coincidentally, these 2 fairways had the lowest and 4th lowest population densities of the 18 that were sampled; not enough grubs were found to discern spatial relationships. Also not included in the analyses were 4 fairways for which the spatial covariance was not strong enough for any of the methods to provide reliable estimates. As a rough measure of reliability, we used the $P$ value of the linear regression of cross-validation estimates against the raw grubs counts. Fairways T1895, T1095, T1295, and S1196 had $P$ values substantially higher than those of the other fairways (Fig. 2), making meaningful comparisons between the methods impossible for these 4 fairways. Therefore, the analysis does not include these fairways.

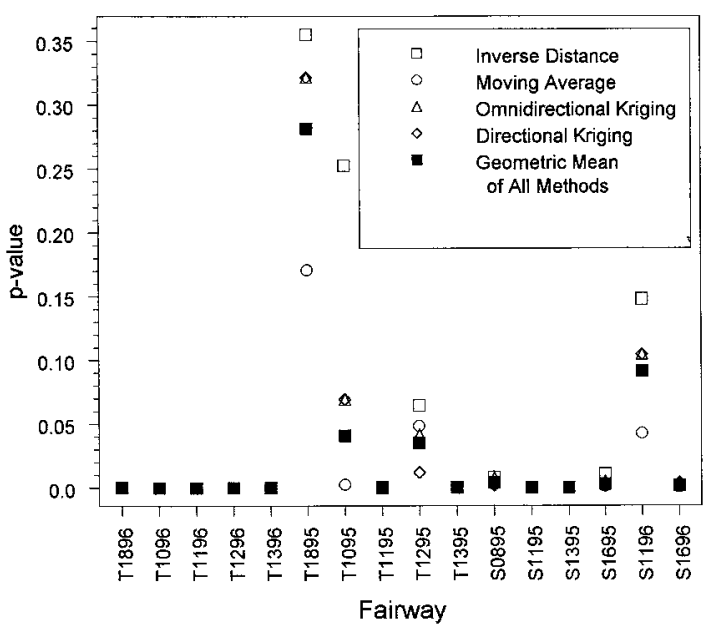

Fig. 2. Fairway-by-fairway $P$ values for linear regression of cross-validation residuals on sample grub counts. For fairways T1895, T1095, T1295, and S1196, the correlations are substantially weaker than they are for the other fairways, indicating that none of the estimation methods is reliable on these data sets.

\section{Results and Discussion}

Effectiveness. The relative effectiveness of the methods (inverse distance, moving average, omnidirectional kriging, directional kriging) was compared on the basis of the MSE and MAE of the cross-validation errors. For each fairway, the inverse distance exponent and moving average radius that minimized the MSE and MAE were calculated and are listed in Table 3. Optimum parameter values varied not only from fairway to fairway but also depended on whether they were optimized with respect to MSE or MAE. For inverse distances and moving averages, the analysis of effectiveness was based on these optimized parameters.

Effectiveness of Moving Averages and Inverse Distances Relative to Directional Kriging. On average, MSE- optimized inverse distances and moving averages were respectively only 82 and $67 \%$ as effective as directional kriging at improving on the null MSE. In Fig. 3 points above the dotted line represent a relative effectiveness $>1$, indicating that the given method is more effective than directional kriging on the fairway represented by the point. Conversely, points below the dotted line indicate that the method is not as effective as directional kriging on the fairway. When compared on the basis of MAE rather than MSE, inverse distances and moving averages were still not as effective as directional kriging, although the differences were less pronounced (geometric means of relative effectiveness were 85 and $90 \%$ for inverse distances and moving averages, respectively).

For all but 1 fairway (S1696), directional kriging was the most effective as measured by MSE. For S1696, inverse distance with an exponent of 2 had a relative effectiveness of 1.35 (optimized for MSE). However, the method's $R^{2}$ was still only 0.047 compared with 
Table 3. Inverse distance and moving average parameters that minimize $M A E$ and $M S E$

\begin{tabular}{|c|c|c|c|c|}
\hline \multirow{2}{*}{ Fairway } & \multicolumn{2}{|c|}{ Inverse distances, exponent: } & \multicolumn{2}{|c|}{ Moving averages, Radius m } \\
\hline & MSE-optimized & $M A E$-optimized & MSE-optimized & $M A E$-optimized \\
\hline T1896 & 1.75 & 2.75 & 15.2 & 8.8 \\
\hline T1096 & 2.25 & 3 & 10.7 & 10.7 \\
\hline T1196 & 2.75 & 3 & 15.2 & 7.6 \\
\hline $\mathrm{T} 1296$ & 2.5 & 3.5 & 10.7 & 10.7 \\
\hline $\mathrm{T} 1396$ & 2 & 2.5 & 18.3 & 8.8 \\
\hline $\mathrm{T} 1195$ & 2.25 & 2.5 & 4.6 & 4.6 \\
\hline $\mathrm{T} 1395$ & 2.25 & 5 & 13.7 & 7.6 \\
\hline S0895 & 1.5 & 2 & 45.7 & 10.7 \\
\hline S1195 & 2 & 3 & 15.2 & 7.6 \\
\hline S1395 & 2 & 1.75 & 13.7 & 13.7 \\
\hline S1695 & 1.25 & 1.5 & 45.7 & 10.7 \\
\hline S1696 & 2 & 4 & 15.2 & 7.6 \\
\hline Mean & 2.04 & 2.88 & 18.67 & 9.09 \\
\hline Critical range & $1.5-2.5$ & $1.75-4$ & $10.7-18.3$ & $7.6-10.7$ \\
\hline
\end{tabular}

directional kriging's 0.035 (Table 4), implying that both methods were only slightly better than the null model in which all local means are assumed to be equal to the overall mean. The relative effectiveness of moving average optimized for MAE is 1.9 for the same fairway, indicating that the method outperformed directional kriging on this fairway. For all other fairways, directional kriging was the most effective with respect to MSE (Fig. 3; Table 4). However, inverse distance and moving average often were much less effective than directional kriging. On 5 of the 12 fairways, MSEoptimized inverse distances were $<75 \%$ as effective as directional kriging, and on 6 fairways MSE-optimized moving averages were $<70 \%$ as effective as directional kriging. On 1 fairway (T1195), MSE-optimized moving averages were outperformed by the null model.

The relative effectiveness of inverse distances and moving averages improved when $M A E$ was the yardstick. The improved performance relative to kriging is

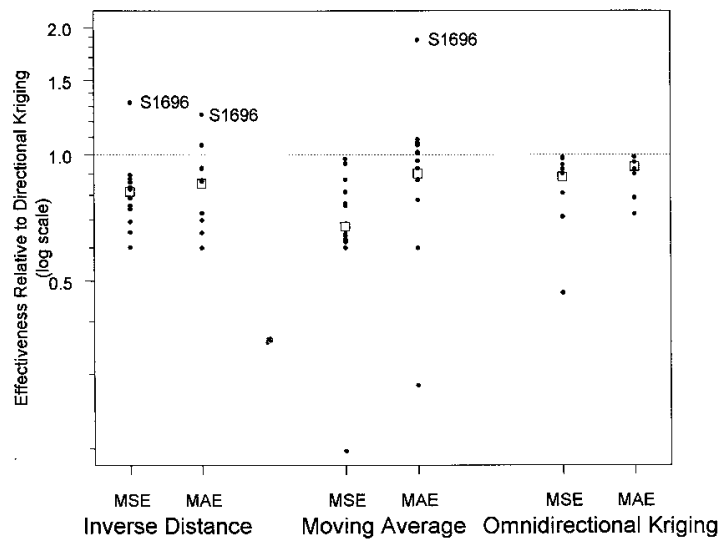

Fig. 3. Relative effectiveness of moving average, inverse distance, and omnidirectional kriging using directional kriging as a standard for comparison. Each dot represents the relative effectiveness of the method indicated on the $\mathrm{x}$-axis for a single fairway. Inverse distance exponents and moving average radii are optimized for MSE and MAE of cross-validation errors, as indicated. Open squares represent geometric means. Points above and below the dotted line represent superiority over and inferiority to directional kriging. not surprising in that inverse distances and moving averages can be optimized to give low $M A E$, whereas kriging inherently minimizes MSE rather than MAE. On S1696, moving averages had substantially higher relative effectiveness than all other methods (Fig. 3). This may be because the low mean population density on the fairway ( 0.23 grubs per sample compared with an average of 1.01 for the other fairways with significant spatial covariance) (Table 1). Only 37 of 225 samples contained grubs. Because the sampling provided so little information about the grub dispersion patterns, the results are highly sensitive to random variation, so the unusually strong performance of moving averages is probably overstated by the relative effectiveness statistic. On 4 other fairways (T1096, T1196, T1296, and T1396) moving averages yielded the lowest MAE of all the methods, although the performances were only slightly better than directional kriging (Fig. 3; Table 4). On all other fairways, directional kriging was still the most effective as measured by $M A E$. On several of these fairways, the differences were large.

In summary, we found that although on some fairways the effectiveness of directional kriging was not significantly better than that of inverse distances or moving averages, directional kriging greatly outperformed the other methods on a third to half of the fairways.

Effectiveness of Omnidirectional Kriging Relative to Directional Kriging. Incorporating the effect of direction into covariogram models when performing directional kriging greatly complicates the modeling effort. In most cases, the added complexity did not result in significantly better estimates of local means, because on most fairways omnidirectional kriging was as effective or nearly as effective as directional kriging. On 9 of the 12 fairways, omnidirectional kriging was $>90 \%$ as effective as directional kriging at improving on the null MSE (Fig 3). On fairways S1695, S0895, and T1396, however, omnidirectional kriging had MSE-based relative effectiveness of $0.48,0.72$, and 0.82 , respectively, indicating that directional kriging was substantially more effective on these fairways. As measured by $M A E$, the performance gap between omnidirectional 
Table 4. Effectiveness of methods of estimating local mean populations

\begin{tabular}{|c|c|c|c|c|c|c|c|c|}
\hline & \multicolumn{4}{|c|}{ Cross-validation $R^{2}$} & \multicolumn{4}{|c|}{ Cross-validation $A$} \\
\hline & $\mathrm{ID}^{a}$ & $\mathrm{MA}^{b}$ & $\mathrm{OK}^{c}$ & $\mathrm{DK}^{d}$ & $\mathrm{ID}^{a}$ & $\mathrm{MA}^{b}$ & $\mathrm{OK}^{c}$ & $\mathrm{DK}^{d}$ \\
\hline T1896 & 0.125 & 0.144 & 0.142 & 0.163 & 0.142 & 0.158 & 0.156 & 0.161 \\
\hline T1096 & 0.157 & 0.175 & 0.177 & 0.177 & 0.133 & 0.168 & 0.152 & 0.152 \\
\hline T1196 & 0.219 & 0.199 & 0.220 & 0.240 & 0.168 & 0.195 & 0.174 & 0.191 \\
\hline T1296 & 0.213 & 0.207 & 0.213 & 0.213 & 0.194 & 0.210 & 0.194 & 0.194 \\
\hline T1396 & 0.124 & 0.137 & 0.145 & 0.177 & 0.090 & 0.126 & 0.114 & 0.122 \\
\hline T1195 & 0.103 & -0.020 & 0.164 & 0.164 & 0.055 & 0.026 & 0.090 & 0.090 \\
\hline T1395 & 0.169 & 0.161 & 0.210 & 0.210 & 0.158 & 0.158 & 0.148 & 0.148 \\
\hline S0895 & 0.102 & 0.086 & 0.098 & 0.136 & 0.046 & 0.042 & 0.055 & 0.069 \\
\hline S1195 & 0.129 & 0.101 & 0.148 & 0.154 & 0.107 & 0.100 & 0.106 & 0.113 \\
\hline S1395 & 0.200 & 0.138 & 0.226 & 0.226 & 0.122 & 0.103 & 0.130 & 0.130 \\
\hline S1695 & 0.069 & 0.065 & 0.049 & 0.104 & 0.048 & 0.063 & 0.049 & 0.067 \\
\hline S1696 & 0.047 & 0.023 & 0.035 & 0.035 & 0.090 & 0.125 & 0.066 & 0.066 \\
\hline
\end{tabular}

${ }^{a}$ Inverse distance weighting.

${ }^{b}$ Moving averages.

${ }^{c}$ Omnidirectional kriging.

${ }^{d}$ Directional kriging.

and directional kriging was smaller than when measured by MSE (Fig. 3). On 10 of the 12 fairways, the relative effectiveness of omnidirectional kriging exceeded $90 \%$ as measured by improvement in MAE over the null model.

Stability. The main advantage that inverse distances and moving averages have over kriging is ease of calculation. We found that, when optimized, inverse distances and moving averages were sometimes comparable to directional kriging with respect to effectiveness. However, optimization is calculationintensive and thus partially negates the advantage of simplicity. Therefore, as others have noted (e.g., Weisz et al. 1995a, b), it would be highly beneficial to be able to use a single, standard parameter that would be applicable to all fields. However, as discussed below, we found that such standardization would contribute substantially to the uncertainty of the estimates made by inverse distances and moving averages.

Stability Index for Moving Averages and Inverse Distances. We quantified the degree of uncertainty associated with choosing a standard inverse distance exponent or moving average radius for use on all fairways by use of the stability index (Table 3 ). An unstable method is not amenable to standardization because the standard parameter is likely to differ enough from the optimum to have a significant impact on the resulting estimates of local means.

Moving averages were more stable than inverse distances on the fairways we sampled (Fig. 4). The stability index for MSE-optimized inverse distances exceeded 3.0 on 11 of 12 fairways, whereas the index for MSE-optimized moving averages was $<3.0$ for 11 of the 12 fairways. The differences in stability between inverse distances and moving averages optimized for $M A E$ were even greater. The stability index for MAEoptimized inverse distances exceeded 3.0 for all 12 fairways, whereas the stability index for MAE-optimized moving averages was $<1.8$ for all 12 fairways. Thus, the method of inverse distances was less well suited for standardization than was moving averages.

Not only was the method of inverse distances less well suited for standardization than was moving av- erages, we also found the method to be unsuitable in an absolute sense. The argument relies on a more detailed discussion of the practical significance of the stability index. Because of space limitations, we limit the detailed discussion to 1 fairway. Fairway T1896 with inverse distances with MSE optimization was selected because it has a stability index of 3.44, which marks a cutoff that separates inverse distances from moving averages with respect to stability (Fig. 4). T1896 is among the most stable fairways for inverse distances, yet its stability index value of 3.44 for MSE is higher than the index for moving averages on every fairway (Fig. 4; Table 5).

Stability Index and Uncertainty of Topography of the Grub Density Map. A stability index of 3.44 corresponds to a large degree of uncertainty about the roughness of the estimated grub density surface, as shown in Fig. 5a and b However, an index of 3.44 is close to the minimum for inverse distances among all fairways. The density surfaces for most of the fairways

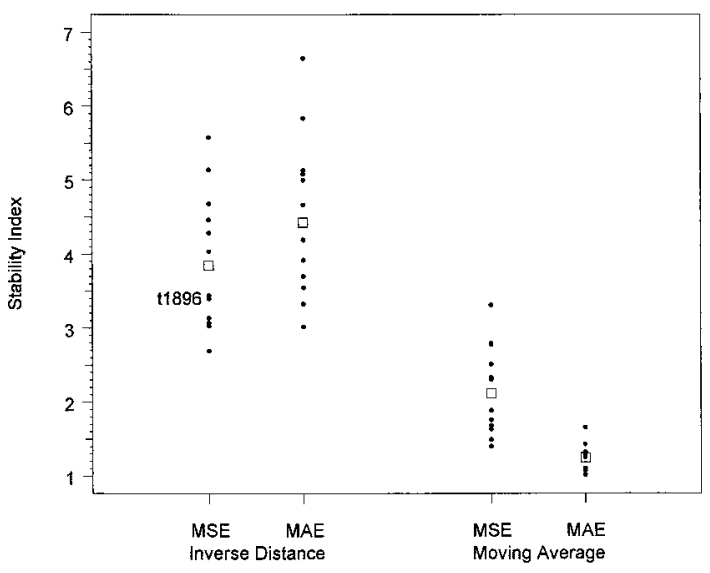

Fig. 4. Stability index for moving averages and inverse distances. Each dot represents the stability index for a single fairway and a given method ( $x$-axis). The value of the index increases with uncertainty about the smoothness of the grub population density surface. 
Table 5. Estimated population variance and stability index (ratio)

\begin{tabular}{|c|c|c|c|c|c|c|c|c|c|c|c|c|}
\hline \multirow{3}{*}{ Fairway } & \multicolumn{6}{|c|}{ Inverse distance } & \multicolumn{6}{|c|}{ Moving avg } \\
\hline & \multicolumn{3}{|c|}{ MSE } & \multicolumn{3}{|c|}{ MAE } & \multicolumn{3}{|c|}{ MSE } & \multicolumn{3}{|c|}{ MAE } \\
\hline & $\mathrm{p}=1.5$ & $\mathrm{p}=2.5$ & $\overline{\text { Ratio }}$ & $\mathrm{p}=1.75$ & $\mathrm{p}=4$ & $\overline{\text { Ratio }}$ & $\mathrm{r}=10.7$ & $\mathrm{r}=18.8$ & $\overline{\text { Ratio }}$ & $\mathrm{r}=7.6$ & $\mathrm{r}=01.7$ & Ratio \\
\hline T1896 & 0.168 & 0.578 & 3.441 & 0.249 & 1.054 & 4.228 & 0.314 & 0.205 & 1.529 & 0.446 & 0.314 & 1.421 \\
\hline T1096 & 0.146 & 0.657 & 4.500 & 0.241 & 1.248 & 5.170 & 0.381 & 0.229 & 1.665 & 0.543 & 0.381 & 1.427 \\
\hline T1196 & 0.420 & 1.332 & 3.170 & 0.599 & 2.368 & 3.955 & 0.790 & 0.549 & 1.439 & 1.116 & 0.790 & 1.412 \\
\hline T1296 & 0.076 & 0.393 & 5.183 & 0.132 & 0.774 & 5.873 & 0.241 & 0.135 & 1.789 & 0.348 & 0.241 & 1.443 \\
\hline T1396 & 0.140 & 0.570 & 4.072 & 0.218 & 1.115 & 5.121 & 0.317 & 0.185 & 1.716 & 0.474 & 0.317 & 1.494 \\
\hline T1195 & 0.270 & 1.170 & 4.324 & 0.454 & 2.133 & 4.702 & 1.028 & 0.307 & 3.348 & 1.738 & 1.028 & 1.691 \\
\hline T1395 & 0.177 & 0.614 & 3.476 & 0.273 & 1.021 & 3.737 & 0.662 & 0.234 & 2.829 & 0.873 & 0.662 & 1.318 \\
\hline S0895 & 0.381 & 1.039 & 2.728 & 0.539 & 1.646 & 3.056 & 1.081 & 0.424 & 2.550 & 1.393 & 1.081 & 1.289 \\
\hline S1195 & 0.131 & 0.618 & 4.720 & 0.226 & 1.141 & 5.042 & 0.636 & 0.272 & 2.336 & 0.933 & 0.636 & 1.467 \\
\hline S1395 & 0.408 & 1.249 & 3.066 & 0.600 & 2.020 & 3.368 & 1.369 & 0.579 & 2.365 & 1.808 & 1.369 & 1.321 \\
\hline S1695 & 0.171 & 0.530 & 3.104 & 0.249 & 0.895 & 3.588 & 0.560 & 0.199 & 2.812 & 0.758 & 0.560 & 1.355 \\
\hline S1696 & 0.027 & 0.153 & 5.618 & 0.048 & 0.320 & 6.684 & 0.058 & 0.030 & 1.919 & 0.102 & 0.058 & 1.767 \\
\hline Mean & 0.210 & 0.742 & 3.950 & 0.319 & 1.311 & 4.544 & 0.620 & 0.279 & 2.192 & 0.878 & 0.620 & 1.451 \\
\hline Geometric mean & - & - & 3.854 & - & - & 4.428 & - & - & 2.117 & - & - & 1.444 \\
\hline
\end{tabular}

vary even more markedly for parameter values in the critical ranges ( $M S E$ and $M A E$ ) for inverse distances. By contrast, Fig. 5c and d illustrate the uncertainty associated with a stability index of 1.53, the value for MSE-optimized moving averages on the fairway. Thus, the moving average surfaces are relatively consistent for all radii in the critical range. The stability index is a general quantification of the differences visible in

A.

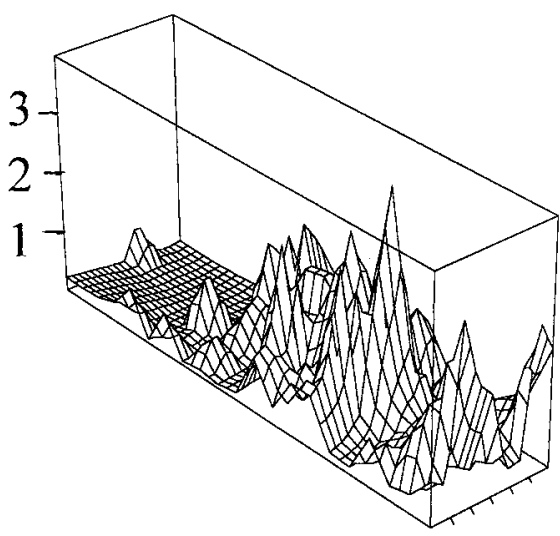

C.

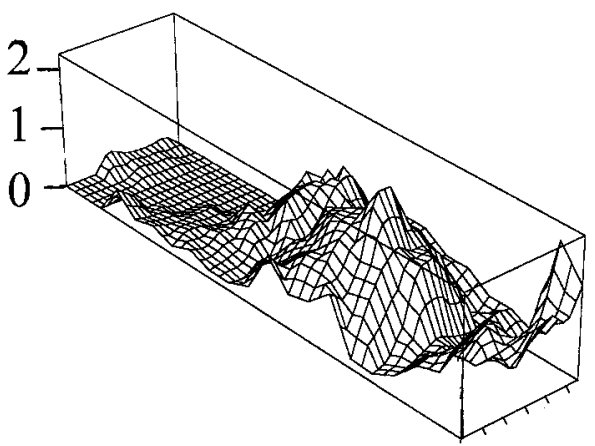

Fig. 5, and as such, the index measures the relative stability of the methods. However, the practical implications of differences in stability are not captured directly by the index. Instead, we examined the stability of the distribution of local means within the critical region for a quantitative assessment of the practical implications of a stability index value of 3.44 .

B.
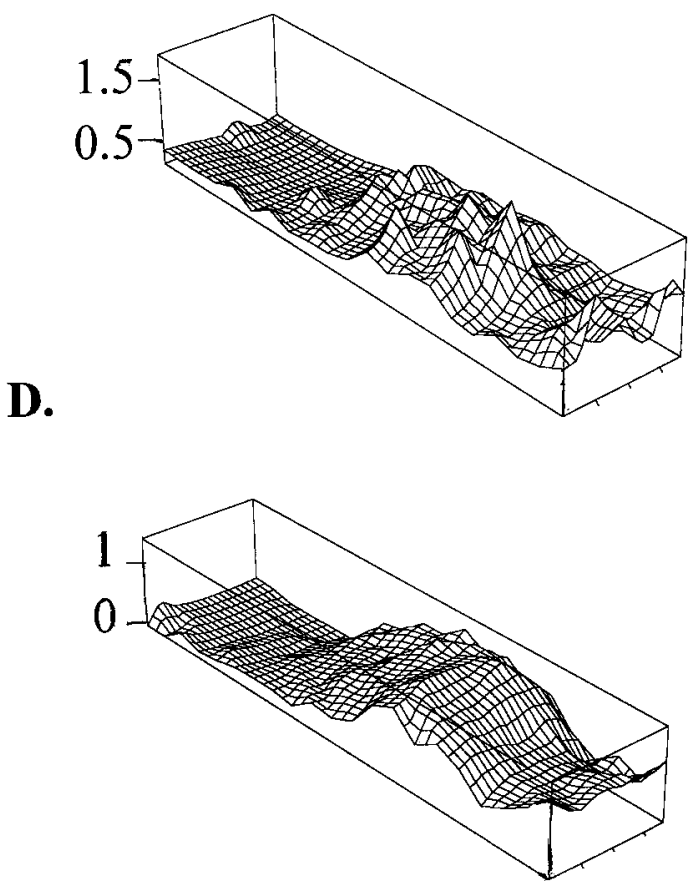

Fig. 5. Estimated grub population density on fairway T1896 using inverse distances and moving averages with parameter values in the critical range. (A) Inverse distance with exponent of 2.5. (B) Inverse distance with exponent of 1.5. (C) Moving average with radius of $10.7 \mathrm{~m}$. (D) Moving average with radius of $18.3 \mathrm{~m}$. 


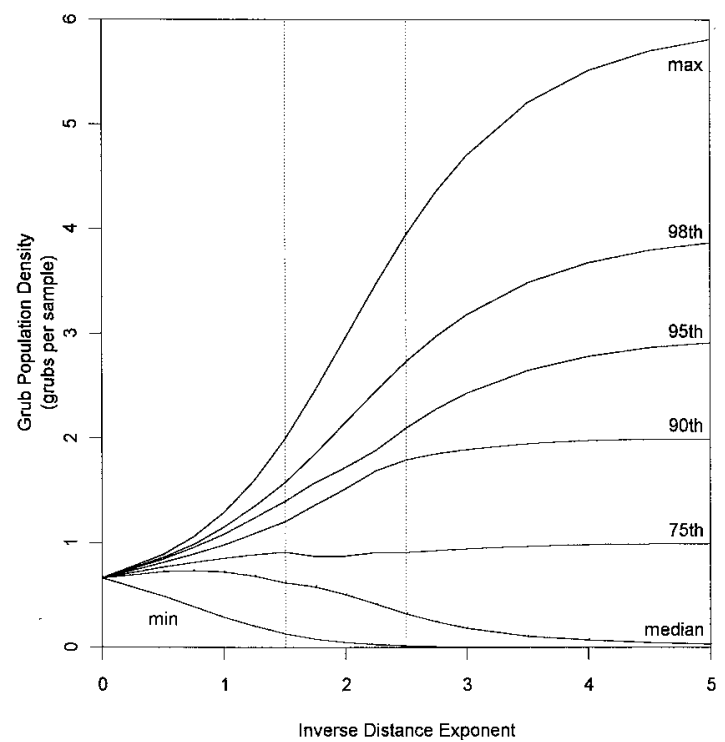

Fig. 6. Percentiles of estimated local mean grub densities on fairway T1896 as functions of inverse distance exponent. Vertical lines represent the boundaries of the critical parameter range.

Stability Index and Uncertainty of Patch Density. The overall mean grub density for the surfaces in Fig. 5a andb is essentially the same- -0.676 for exponent 1.5 and 0.667 for exponent 2.5. The differences between the surfaces lie in the heights of the peaks and the depths of the troughs. The sizes of the peaks can be compared via the upper tails of the distributions of local means. In Fig. 6, a given percentile of estimated local grub density is plotted against inverse distance exponent $p$. The lowest line is the estimated minimum grub density. Lines for the median, 75th, 90th, 95th, and 98th percentiles are above that, with the line for the maximum on top. For $p=0$, all local means are equal to the overall sample mean, so the lines start at the local mean and spread out as $p$ increases. Steepness in the curve is indicative of rapid change in grub density surface corresponding to relatively small changes in exponent. For example, with an exponent of 1.5 , the estimated maximum population density is 1.99 , but for an exponent of 2.5 , the expected maximum rises to 3.94 , nearly twice as high. Thus, within the critical range for MSE, estimates of the maximum grub density on the fairway vary by nearly a factor of 2 , making inverse distances unreliable for estimating the maximum population density on this fairway.

Stability Index and Uncertainty About Patch Extent. A turfgrass manager interested in treating only those sections of the fairway that harbor grub patches with density exceeding a threshold would need to identify the boundaries of those patches. A commonly used treatment threshold for Japanese beetle grubs in turfgrass is 100 grubs per square meter, or $\approx 1$ per sample. According to inverse distance with an exponent of 1.5, $21 \%$ of the fairway (T1896) has a density exceeding 1 and would be treatable. With an exponent of 2.5 , the

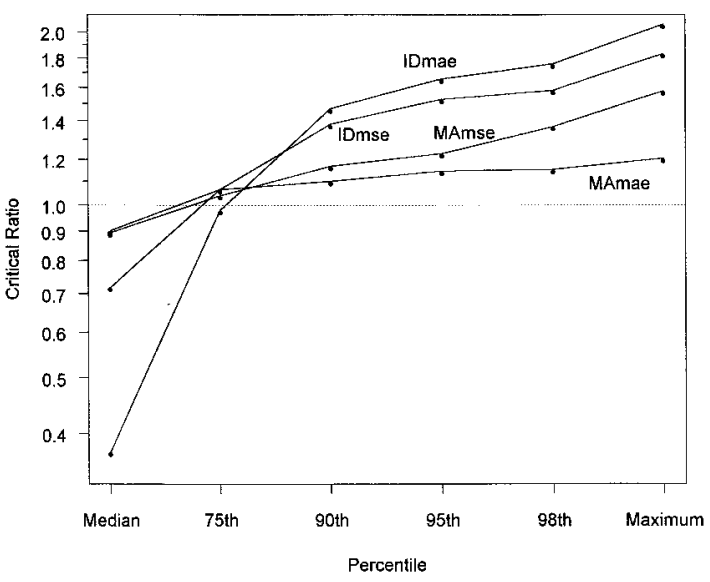

Fig. 7. Stability of smoothing methods with respect to distribution of local means. A critical ratio near 1.0 indicates that the method gives consistent estimates of the given percentile for all parameters in the critical range. ID, method of inverse distances; MA, moving averages; mae and mse denote which critical range (MSE or MAE) was used to calculate the critical ratios. Critical ratios are the mean critical ratios across all fairways.

treatable fraction of the fairway rises only to $23 \%$. This indicates that for T1896, choice of exponent within the critical range has very little effect on the estimation of the treatable portion of the fairway given a treatment threshold of 1 grub per sample, making inverse distance a seemingly reliable tool. For a treatment threshold of 1.5 , however, the situation changes dramatically. An exponent of 1.5 results in an estimate of $2.8 \%$ of the fairway requiring treatment. An exponent of 2.5 increases the estimated treatable portion of the fairway to $17 \%$, a 6 -fold increase, making inverse distance an unreliable tool for identifying patch boundaries. Fig. 6 suggests that inverse distance gives a consistent estimate of the 75th percentile of grub density on T1896. However, it gives inconsistent estimates of the upper quantiles of population density. Thus, a stability index of 3.44 or higher is a strong indication that the method may be unreliable for many types of ecological problems. Because the stability index was commonly in excess of 3.44 for inverse distances (both for MSE and $M A E$ ), the method was not suitable for standardization by using a single exponent for all fairways.

Suitability of Standardization of Inverse Distance and Moving Averages. As alluded to in the previous paragraph, suitability for standardization is dependent upon the question of interest. Fig. 7 shows how the upper tail of the distribution of estimated local means (i.e., the heights of the peaks) varies for parameters in the critical range. We defined the critical ratio for a method as the ratio of the estimates of a given grub density percentile for parameters on the boundaries of the critical range. The critical ratio was calculated for each fairway for inverse distances and moving averages for a number of percentiles. The average critical ratio in Fig. 7 is the geometric mean of the ratios for all the fairways, and it is a measure of how stable a 
given method is for estimating a given quantile on average. Thus, Fig. 7 shows that the least stable method on average is inverse distance optimized for $M A E$. Within the critical parameter range for this method, estimates of the median vary by a factor of 2.7 , which is far higher than for any other method. All the methods reliably estimate the 75 th percentile on average, but the critical ratio for $M A E$-optimized inverse distance estimates is 1.5 or more for the 90th percentile and above and exceeds all other methods at every percentile higher than the $75 \mathrm{th}$. The critical ratios for moving averages (optimized for either MSE or MAE) are between 0.8 and 1.25 for all quantiles between the median and 95th percentile. The most stable method is moving averages optimized for $M A E$-all critical ratios are between 0.8 and 1.25.

In summary, without optimization, the inverse distance method was too unstable to reliably estimate the median population density, upper percentiles (or heights of the peaks on the grub density maps), or maximum local means. Even when optimized for MSE or MAE, cross-validation indicates that the inverse distance method is less effective than directional kriging at estimating local means.

The method of moving averages was more stable than inverse distances; it may even be stable enough for a standard radius to be used for some applications, especially when the standard is based on the minimization of MAE. Moving averages optimized for MSE was considerably less effective than either directional or omnidirectional kriging; but when optimized for $M A E$, it was often comparable in effectiveness to omnidirectional kriging.

Directional kriging consistently gave better estimates of local means than either moving averages or inverse distances when measured by MSE. When measured by $M A E$, moving averages gave slightly better estimates than directional kriging on some fairways, but on $25 \%$ of the fairways, it was substantially outperformed by directional kriging.

Natural variation in the spatial patterns of grub abundance from fairway to fairway and year to year was great enough that selection of a standard exponent for inverse distances or a standard smoothing radius for moving averages often resulted in poor estimates of local means, especially in sections of fairways where grub density was relatively high. When the variation in grub patterns was implicitly accounted for by optimization of exponent or smoothing radius on a fairway-by-fairway basis, the stability and effectiveness of moving averages and inverse distances improved, but only with an increase in complexity. Moreover, even with optimization, inverse distances and moving averages were still far less effective than kriging for several fairways.

In conclusion, we demonstated that in some situations standardized inverse distances or moving averages may perform just as well or nearly as well as directional kriging, whereas for other situations, the methods may be vastly outperformed by kriging, even after they are optimized. Their performance on a particular data set depends on whether the spatial covariance of the data by chance happens to be compatible with the inverse distance or moving average model. Therefore, inverse distances and moving averages tend to be unreliable, even though they occasionally may be just as effective as directional kriging. Because kriging is linked to the ecology of the organism via an explicit model of the spatial covariance, it naturally adjusts itself to the spatial patterns in the data and therefore performs more consistently and reliably than inverse distances or moving averages. Because of its higher degree of reliability, kriging is to be recommended over inverse distances or moving averages for estimation of local means.

\section{Acknowledgments}

We thank Nancy Consolie for stellar technical assistance in the field, Lisa Madsen for statistical advice and assistance, and Dave Benarski and Norm Sharman for their generous cooperation in allowing us to sample their golf courses. This study was supported by funds from USDA-NRI, Northeast Regional Integrated Pest Management (IPM), NY State IPM, USGA, Musser International Turfgrass Foundation, and the Cornell University Departments of Entomology (Geneva and Ithaca, NY).

\section{References Cited}

Cressie, N.A.C. 1993. Statistics for spatial data. Wiley, New York.

Deutsch, C. V., and A. G. Journel. 1992. GSLIB: geostatistical software library and user's guide. Oxford University Press, New York.

Isaaks, E. H., and R. M. Srivastava. 1989. Applied geostatistics. Oxford University Press, New York.

Kaluzny, S. P., S. C. Vega, T. P. Cardoso, and A. A. Shelly. 1996. S+SpatialStats user's manual. MathSoft, Seattle, WA.

Midgarden, D., S. J. Fleischer, R. Weisz, and Z. Smilowitz. 1997. Site-specific integrated pest management impact on development of esenvalerate resistence in Colorado potato beetle (Coleoptera: Chrysolmelidae) and on densities of natural enemies. J. Econ. Entomol. 90: 855-867.

Nyrop, J. P., M. G. Villani, and J. A. Grant. 1995. Control decision rule for European chafer (Coleoptera: Scarabaeidae) larvae infesting turfgrass. Environ. Entomol. 24: 521-528.

Weisz, R., S. Fleischer, and Z. Smilowitz. 1995a. Site-specific integrated psest management for high value crops: sample units for map generation using the Colorado potato beetle (Coleoptera: Chrysomelidae) as a model system. J. Econ. Entomol. 88: 1069-1080.

1995b. Map generation in high-value horticultural integrated pest management: appropriate interpolation methods for site-specific pest management of Colorado potato beetle (Coleoptera: Chrysolmelidae). J. Econ. Entomol. 88(6): 1650-1657.
Received for publication 17 April 1998; accepted 23 October 998. 


\section{Appendix 1: Determination of Inverse Distance Weights for Center Points of Blocks.}

Because samples are taken at the center of each block, an inverse distance weighting would give each sample infinite weight in the determination its block mean. The estimates of the block means would simply be the raw sample values. Because the problem is to estimate the mean in a small area, a possible alternative would be to make conventional, interpolated inverse distance estimates for a number of points in each block. The block mean estimate would be then be the average of the interpolated point estimates. This proposed solution has 2 drawbacks as follows: (1) it substantially increases the difficulty of the computations in terms of computer memory and CPU time; (2) $\lim _{\min \left|x-x_{0}\right| \rightarrow 0}\left(\hat{\mu}_{o}\right)=v_{0}$, or as more and more points are averaged, the estimated mean approaches the raw sample count. The 1st drawback is overcome by noting that averaging a finite number of interpolated points in a block is equivalent to assigning a proper weight to the central point. The ideal weight varies with the number of interpolated points, the size of the block, and the inverse distance exponent. Problem (2) is overcome by taking a moderate number of interpolated points for each block size. For powers between 0.5 and 5 and for an equally spaced grid of 16 interpolated points within a block, the ideal weight is be-
Table 2. Weights assigned to samples at centers of blocks for inverse distance method

\begin{tabular}{lcc}
\hline \hline \multirow{2}{*}{ Power } & \multicolumn{2}{c}{ Weight $^{a}$} \\
\cline { 2 - 3 } & 4.6 by $7 \mathrm{~m}$. grid & 4.6 by $14 \mathrm{~m}$. grid \\
\hline 0.5 & 0.3901 & 0.3074689 \\
0.75 & 0.2437 & 0.170491 \\
1 & 0.1522 & 0.094537 \\
1.25 & 0.09507 & 0.052421 \\
1.5 & 0.05938 & 0.029067 \\
1.75 & 0.03709 & 0.016118 \\
2 & 0.02317 & 0.0089373 \\
2.25 & 0.01447 & 0.004956 \\
2.5 & 0.009038 & 0.002748 \\
2.75 & 0.005645 & 0.001523 \\
3 & 0.003526 & 0.00084490 \\
3.5 & 0.001376 & 0.0002598 \\
4 & 0.0005367 & 0.00007987 \\
4.5 & 0.0002094 & 0.00002456 \\
5 & 0.00008169 & 0.000007551 \\
\hline
\end{tabular}

${ }^{a}$ Weights are the $w_{\mathrm{j}}$ in equation 1.

tween the arithmetic mean and the harmonic mean of the weights that assigned to the 16 points by their inverse distance to the center of the block. The ideal weight never varies far from the geometric mean, so that is the weight we use throughout the study. Thus, the preliminary weights given to individual central samples in the estimation of the mean density in their respective blocks are given in Table 2 (these weights are the $w_{\mathrm{j}}^{\prime} \mathrm{s}$ in equation 1 ). 